12. Mullins, R.F., Russell, S.R., Anderson, D.H., and Hageman, G.S. 2000. Drusen associated with aging and age-related macular degeneration contain proteins common to extracellular deposits associated with atherosclerosis, elastosis, amyloidosis, and dense deposit disease. FASEB J. 14:835-846.

13. Ambati, J., et al. 2003. An animal model of agerelated macular degeneration in senescent Ccl-2- or Ccr-2-deficient mice. Nat. Med. 9:1390-1397.

14. Apte, R.S., Richter, J., Herndon, J., and Ferguson, T.A. 2006. Macrophages inhibit neovascularization in a murine model of age-related macular degeneration. PLoS Med. 3:e310.

15. Espinosa-Heidmann, D.G., et al. 2003. Macrophage depletion diminishes lesion size and severity in experimental choroidal neovascularization. Invest. Ophthalmol. Vis. Sci. 44:3586-3592.

16. Arnold, L., et al. 2007. Inflammatory monocytes recruited after skeletal muscle injury switch into antiinflammatory macrophages to support myogenesis. J. Exp. Med. 204:1057-1069.

17. 2001. A randomized, placebo-controlled, clinical trial of high-dose supplementation with vitamins $\mathrm{C}$ and $\mathrm{E}$, beta carotene, and zinc for age-related macular degeneration and vision loss: AREDS report no. 8. Arch. Ophthalmol. 119:1417-1436.

18. Beatty, S., Koh, H., Phil, M., Henson, D., and Boulton, M. 2000. The role of oxidative stress in the pathogenesis of age-related macular degeneration. Surv. Ophthalmol. 45:115-134.

19. Seddon, J.M., Cote, J., Page, W.F., Aggen, S.H., and Neale, M.C. 2005. The US twin study of age-related macular degeneration: relative roles of genetic and environmental influences. Arch. Ophthalmol. 123:321-327.

20. Teupser, D., et al. 2004. Major reduction of atherosclerosis in fractalkine (CX3CL1)-deficient mice is at the brachiocephalic artery, not the aortic root. Proc. Natl. Acad. Sci. U. S. A. 101:17795-17800.

21. Lavergne, E., et al. 2003. Fractalkine mediates natural killer-dependent antitumor responses in vivo. Cancer Res. 63:7468-7474

\title{
Why targeted therapy hasn't worked in advanced cancer
}

\author{
Jack L. Arbiser \\ Department of Dermatology and Winship Cancer Institute, Emory University School of Medicine, and Atlanta Veterans Administration Medical Center, \\ Atlanta, Georgia, USA.
}

\begin{abstract}
In this issue of the JCI, Nissen et al. report that a reciprocal interaction exists between the growth factors FGF2 and PDGF-BB, causing tumors to exhibit increased angiogenesis and metastatic potential (see the related article beginning on page 2766). Both FGF2 and PDGF-BB signal through tyrosine kinase receptors, which have been the target of tyrosine kinase inhibitors for cancer therapies. These inhibitors are usually small molecules that inhibit the kinase activity of a receptor or nonreceptor tyrosine kinase, preventing downstream signaling. The results of this study shed light on why tyrosine kinase inhibitors have been useful for the treatment of only a small number of advanced cancers. Currently, a major focus of pharmaceutical companies is to develop ever more potent and specific tyrosine kinases. The results presented here suggest that this approach may not be successful.
\end{abstract}

Tyrosine kinases are a large family of enzymes that phosphorylate target proteins, resulting in either activation or inactivation of these proteins. This family includes many peptide receptors as well as nonreceptor proteins and is well represented in oncogenic fusion proteins, such as the BCR-ABL protein, which is generated from the Philadelphia chromosome. These proteins activate multiple signaling pathways, including those involving PI3K, phospholipase $\mathrm{C} \gamma$, MAPK, and STAT activation and the generation of reactive oxygen species. The study by Nissen et al. in this issue of the JCI demonstrates that activation of a combination of several tyrosine kinase receptors results in a high-

Nonstandard abbreviations used: Ang-2, angiopoietin 2; CML, chronic myelogenous leukemia; FGFR1, FGF receptor 1 .

Conflict of interest: The author has declared that no conflict of interest exists.

Citation for this article: J. Clin. Invest. 117:2762-2765 (2007). doi:10.1172/JCI33190. ly angiogenic and metastatic phenotype and that the functions of these tyrosine kinase receptors is nonredundant in vivo (1). These findings may help explain why tyrosine kinases have been successful primarily in early malignancies but less so in advanced cancers.

In the first decade of the twenty-first century, the focus in the field of oncology has been on developing of targeted therapy against tyrosine kinases. This began with the use of the tyrosine kinase inhibitor imatinib, which has shown impressive activity against chronic myelogenous leukemia (CML), and has subsequently been successful in the treatment of other malignancies including gastrointestinal stromal tumors and mastocytosis (2). Other specific tyrosine kinase inhibitors have since appeared, including sorafenib, which was designed to inhibit the mutant $B$-raf oncogene in melanoma (3), in addition to both small molecule and antibody-mediated inhibitors of EGFR (4). EGFR, a member of the tyrosine kinase receptor family, is expressed in a wide variety of epithelial tumors in humans, including breast cancer and non-small cell carcinoma of the lung, calling attention to it as a druggable target. More recently, clinical results have identified some of the shortcomings of such targeted therapies. While imatinib is highly effective against early CML, it is less effective against blast crisis CML (2). Sorafenib was found to be relatively ineffective against melanoma in clinical trials (3), and EGFR tyrosine kinase inhibitors have been found to be most efficacious only in a small subset of patients - often female Japanese nonsmokers with bronchoalveolar histology $(4,5)$. While one response to these failures has been sustained efforts to identify more potent inhibitors, the study by Nissen et al. (1) should give drug developers pause. These authors show that it is the nonredundancy of tyrosine kinases such as FGF receptor 1 (FGFR1) and PDGFR, rather than the potency of the tyrosine kinase inhibitor, that is the main enemy of targeted therapy in cancer. Yet there is room for optimism if one uses combination or sequential tyrosine kinase inhibitor therapy instead of targeted monotherapy. Thus a "dirtier" approach to tyrosine kinase therapy is suggested, referring to the use of both promiscuous tyrosine kinase inhibitors, which inhibit more than one tyrosine kinase as well as a combination of tyrosine kinase inhibitors, and other signaling inhibitors, such as rapamycin. 


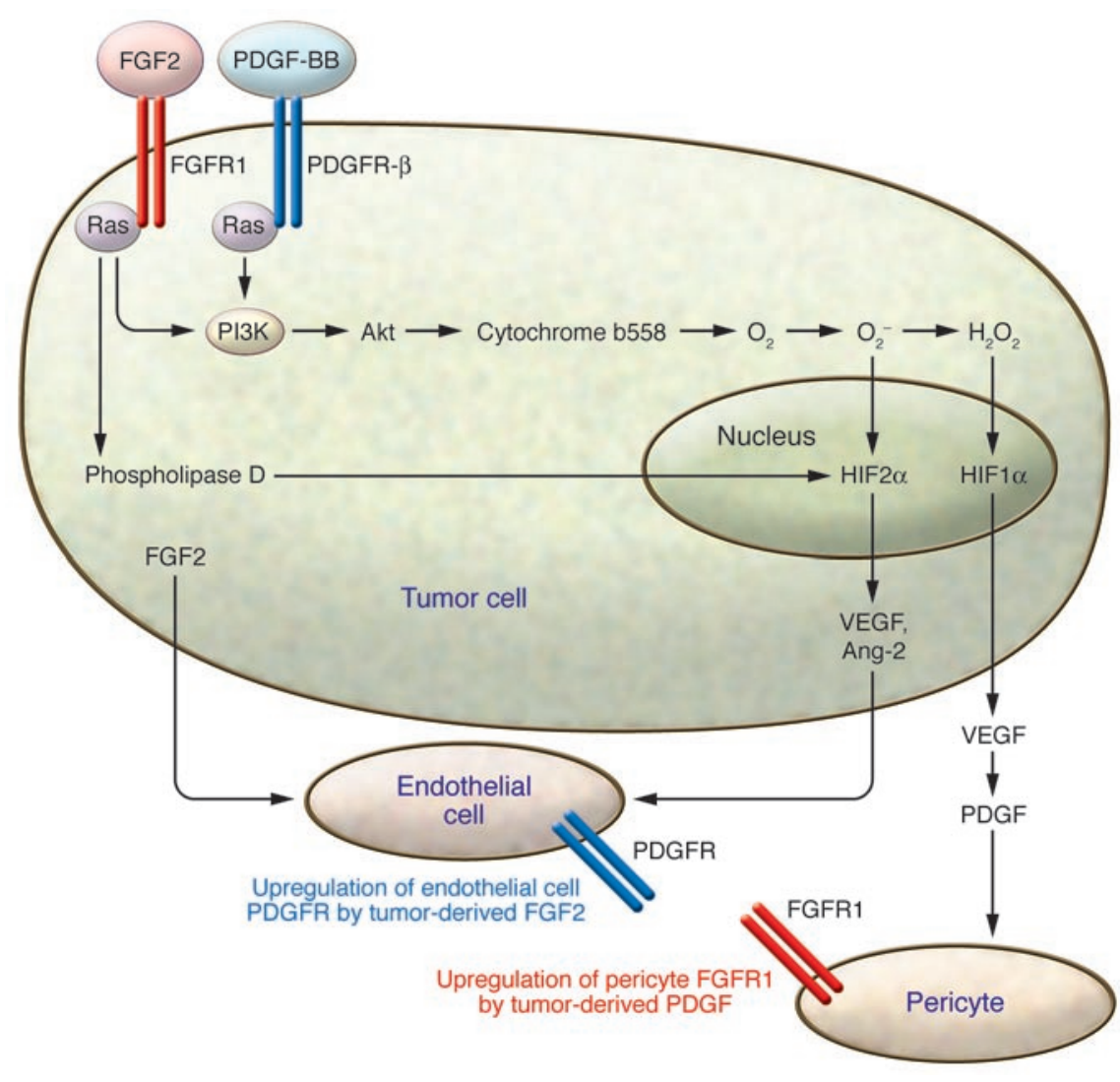

\section{Demonstration of synergy between multiple receptor tyrosine kinases}

Folkman was the first to hypothesize that tumors produce proangiogenic factors, which promote local tumor growth, invasion, and metastasis (6). This observation, in addition to the ability to culture microvascular endothelial cells, led to the purification of the first known angiogenic factors, FGF2 (also known as basic FGF) and VEGF, both of which signal through tyrosine kinase receptors (6). It has been assumed that tumors synthesize multiple angiogenic and growth factors, but this production has been assumed to be a redundancy that only becomes functional if a tumor is challenged with an inhibitor of a specific tyrosine kinase, in which case the tumor can display resistance by switching its dependence to an alternate growth/ angiogenic factor. The findings reported by Nissen et al. (1) demonstrate that tyrosine kinases play a nonredundant role in the stimulation of angiogenesis and metastasis in normal tumor physiology, even when tumors are not challenged by tyrosine kinase inhibitors. Furthermore, the coexpression of two growth factors (FGF2 and PDGF-BB) in mice was shown to confer properties not seen with overexpression of either growth factor independently. While FGF2 and PDGF-BB signal through tyrosine kinases that show activation similar to PI3K and Ras, these growth factors may show differential activation of molecules in downstream signaling pathways, including reactive oxygen species, Akt, and phospholipase D $(7,8)$. In certain circumstances, FGF2 stimulates phospholipase D strongly and Akt poorly, while PDGF-BB is a potent activator of Akt (9-11).

In their study, Nissen et al. (1) have elegantly shown a synergy in vitro and in vivo between PDGF-BB and FGF-2 in the stimulation of angiogenesis, recruitment of an embryonic vascular phenotype, and an enhancement of metastasis. First, in murine corneal neoangiogenesis studies, the coimplantation of FGF2 and PDGFBB led to tumor-like neovascularization, as opposed to the effect of either FGF2 or PDGF-BB alone. Implantation of these growth factors individually resulted in attenuated vessels that rapidly regressed. Second, they demonstrated that FGF2 induces both the transcription of PDGF$\mathrm{BB}$ in endothelial cells and the elevations of phosphorylated MAPK and phospholipase $C \gamma$, both of which are required for optimal tumorigenesis. Third, murine

\section{Figure 1}

Coexpression of FGF2 and PDGF-BB results in activation of nonredundant signaling pathways in advanced solid tumors (1). FGF2 and PDGF-BB both activate tyrosine kinase receptors (FGFR1 and PDGFR- $\beta$, respectively) that in turn activate PI3K signaling, yet differ in their downstream consequences. PDGF-BB is a well-known stimulator of Akt, which can activate reactive oxygen species (superoxide $\left[\mathrm{O}_{2}{ }^{-}\right]$and hydrogen peroxide $\left.\left[\mathrm{H}_{2} \mathrm{O}_{2}\right]\right)$ through induction of cytochrome b558 genes, resulting in the induction of HIF1 $\alpha$ and HIF2 $\alpha$ and eventually VEGF, a potent endothelial cell chemoattractant, and Ang-2, which likely mediates a repulsive effect that prevents pericyte-endothelial cell stabilization of neoangiogenic vessels. FGF2 is a potent activator of phospholipase $D$, that acts as an alternative survival factor to Akt. Blockade of PDGF by a specific tyrosine kinase inhibitor may promote FGF2 signaling, while blockade of FGF2 signaling may promote PDGF-BB-mediated signaling. Blockade of both FGF2 and PDGF-BB with a promiscuous tyrosine kinase inhibitor or other dirty compound may be more effective than targeted tyrosine kinase inhibitor monotherapy in blocking tumor growth.

fibrosarcoma cells coexpressing FGF2 and PDGF-BB exhibited rapid tumor growth in vivo and the development of primitive vascular plexuses in the tumors, reminiscent of what is observed in human tumors. Surprisingly, the vessels in the tumors were not highly invested with pericytes (which normally envelop and stabilize the vessel exterior). Despite the known chemoattractant effects of PDGF on pericytes (12), pericyte recruitment was inhibited and tumor vessels appeared to be disorganized. Finally, the incidence of pulmonary metastases was increased in tumors coexpressing FGF2 and PDGF-BB in comparison with tumors expressing a single growth factor (1). While the minimum requirements for the transformation of human cells have been elucidated in several tissue types, the rationale for a solid tumor to express multiple growth factors in the absence of selective pressure remains poorly understood.

\section{Advanced tumors activate signaling through several pathways}

Many human tumors overexpress tyrosine kinase receptors, including common epithelial tumors such as those in lung and breast tissue $(13,14)$. While overexpression of growth factors that signal through 
tyrosine kinase receptors is sufficient to transform even human cells (7), breast and lung cancers often demonstrate additional mutations, including mutations in K-ras in lung cancer and loss of PTEN in Her2positive breast cancer, even in the absence of selective pressure $(15,16)$. Indeed, the presence of K-ras mutations in lung cancer predicts a poor response to EGFR antagonists despite the biochemical activity of the antagonist on the relevant receptor $(17,18)$. Several forms of signaling redundancy have been characterized at the biochemical level. First, we have previously observed the common activation of reactive oxygen species (e.g., superoxide, hydrogen peroxide) with Akt and MAPK activation in cell types that have lost the $p 16$ tumor suppressor gene (19-21). These tumors arise in the presence of carcinogenic stimuli that induce reactive oxygen species such as chronic inflammation or viral infection. Reactive oxygen species drive NF- $\mathrm{KB}$ activation and trigger downstream effectors such as the angiogenic growth factor angiopoietin 2 (Ang-2) (Figure 1) (22). Ang-2 may play a critical role in the development of the primitive vascular plexuses observed by Nissen et al. (1) as well as in human tumors. Conversely, cells with mutations in the tumor suppressor gene $p 53$ often do not have increased Akt levels but activate another survival signal, the generation of phospholipase D $(23,24)$. While tumors with mutant p53 express tyrosine kinase receptors, they survive serum deprivation, a potential form of tyrosine kinase inhibition, through activation of phospholipase D and Ras (23).

The resistance of tumor cells to targeted therapy with tyrosine kinase inhibitors, due to the presence of multiple tyrosine kinase receptors or coexpression of tyrosine kinase receptors with oncogenic Ras or mutant PTEN, may appear to be a bleak outcome, but there is room for optimism because the response to targeted therapy is predictable and thus vulnerable. In a previous study of Epstein-Barr virus-induced Burkitt lymphoma, we demonstrated that treatment of these cells with $\mathrm{N}$-acetyl cysteine resulted in an inhibition of NF- $\mathrm{KB}$ as expected, but also in a compensatory activation of p42/44 MAPK (20). While blockade of NF- $\mathrm{B}$ may not have an immediate effect on tumor growth in vitro or in vivo, it may sensitize cells to MAPK inhibition. Similarly, sorafenib has been found to be ineffective against advanced melanoma (3), and we have recently demonstrated that aggressive melanoma involves both MAPK signaling as well as Akt/reactive oxygen species/NF-KB signaling (21). Blockade of reactive oxygen species may make cells more sensitive to MAPK blockade and sensitize cells to sorafenib. Finally, tyrosine kinase blockade in advanced solid tumors with mutant p53 may lead to activation of phospholipase D and sensitize tumor cells to phospholipase D blockade.

Screening by immunohistochemistry for a small panel of markers may allow the oncologist and pathologist to determine optimal therapy. The presence or absence of mutant p53 may help determine whether a tumor uses a reactive oxygen species-dependent or-independent signaling pathway. The presence of Ang-2 may also suggest that a tumor is using a reactive oxygen species-dependent pathway (22). Along with the use of stains already utilized for the detection of tyrosine kinase receptor signaling, both the presence of a mutation and the response to targeted therapy can be determined. Finally, the efficacy of targeted therapy can be improved against advanced malignancies. The current study by Nissen et al. (1) is the first to show a synergistic interaction of 2 growth factors that play a nonredundant role in tumor angiogenesis and metastasis. First, FGF2 and PDGF-BB in combination play a role in organizing tumor neovasculature that is distinct from the effect of the presence of either growth factor alone. A possible mediator of this combined effect may be Ang-2, a growth factor known to antagonize pericyte investment of blood vessels $(25,26)$. Of interest, Ang-2 is known to be a poor prognostic factor in multiple cancers (27, 28). Second, the demonstrated effects of reciprocal interaction of multiple growth factors provides a cautionary note against the development of more potent and more specific tyrosine kinase inhibitors. The data reported here by Nissen et al. suggest that the use of dirtier (i.e., more promiscuous) tyrosine kinase receptors, or a combination of therapy with tyrosine kinase inhibitors and other signaling inhibitors (i.e., rapamycin, farnesyltransferase inhibitors) may be more efficacious in the treatment of cancer than ultraspecific and ultrapotent tyrosine kinase inhibitors.

Address correspondence to: Jack L. Arbiser, Department of Dermatology, Emory University School of Medicine, WMB 5309, 1639 Pierce Drive, Atlanta, Georgia 30322, USA. Phone: (404) 727-5063; Fax: (404) 727-0923; E-mail: jarbise@emory.edu.
1. Nissen, L.J., et al. 2007. Angiogenic factors FGF2 and PDGF-BB synergistically promote murine tumor neovascularization and metastasis. J. Clin. Invest. 117:2766-2777. doi:10.1172/JCI32479.

2. Sawyers, C.L., et al. 2002. Imatinib induces hematologic and cytogenetic responses in patients with chronic myelogenous leukemia in myeloid blast crisis: results of a phase II study. Blood. 99:3530-9.

3. Eisen, T., et al. 2006. Sorafenib in advanced melanoma: a Phase II randomised discontinuation trial analysis. Br. J. Cancer. 95:581-586.

4. Hsieh, R.K., Lim, K.H., Kuo, H.T., Tzen, C.Y., and Huang, M.J. 2005. Female sex and bronchioloalveolar pathologic subtype predict EGFR mutations in non-small cell lung cancer. Chest. 128:317-321.

5. Yatabe, Y., Kosaka, T., Takahashi, T., and Mitsudomi, T. 2005. EGFR mutation is specific for terminal respiratory unit type adenocarcinoma. Am. J. Surg. Pathol. 29:633-639.

6. Folkman, J., Merler, E., Abernathy, C., and Williams, G. 1971. Isolation of a tumor factor responsible for angiogenesis. J. Exp. Med. 133:275-288.

7. Govindarajan, B., et al. 2005. Malignant transformation of human cells by constitutive expression of platelet-derived growth factor-BB. J. Biol. Chem. 280:13936-13943.

8. Arbiser, J.L., Byers, H.R., Cohen, C., and Arbeit, J. 2000. Altered basic fibroblast growth factor expression in common epidermal neoplasms: examination with in situ hybridization and immunohistochemistry. J. Am. Acad. Dermatol. 42:973-977.

9. Cross, M.J., et al. 2000. Tyrosine 766 in the fibroblast growth factor receptor-1 is required for FGFstimulation of phospholipase C, phospholipase D, phospholipase A(2), phosphoinositide 3-kinase and cytoskeletal reorganisation in porcine aortic endothelial cells. J. Cell Sci. 113:643-651.

10. Li, L., Sampat, K., Hu, N., Zakari, J., and Yuspa, S.H. 2006. Protein kinase $\mathrm{C}$ negatively regulates Akt activity and modifies UVC-induced apoptosis in mouse keratinocytes. J. Biol. Chem. 281:3237-3243.

11. Kiss, Z. 1992. Differential effects of platelet-derived growth factor, serum and bombesin on phospholipase D-mediated hydrolysis of phosphatidylethanolamine in NIH 3T3 fibroblasts. Biochem. J. 285:229-233.

12. Hirschi, K.K., Rohovsky, S.A., and D'Amore, P.A. 1998. PDGF, TGF-beta, and heterotypic cell-cell interactions mediate endothelial cell-induced recruitment of $10 \mathrm{~T} 1 / 2$ cells and their differentiation to a smooth muscle fate. J. Cell Biol. 141:805-814.

13. Rodriguez-Pinilla, S.M., et al. 2007. Sox2: a possible driver of the basal-like phenotype in sporadic breast cancer. Mod. Pathol. 20:474-481.

14. Slamon, D.J., et al. 1987. Human breast cancer: correlation of relapse and survival with amplification of the HER-2/neu oncogene. Science. 235:177-182.

15. Nagata, Y., et al. 2004. PTEN activation contributes to tumor inhibition by trastuzumab, and loss of PTEN predicts trastuzumab resistance in patients. Cancer Cell. 6:117-127.

16. van Zandwick, N., et al. 2007. EGFR and KRAS mutations as criteria for treatment with tyrosine kinase inhibitors: retro- and prospective observations in non-small-cell lung cancer. Ann. Oncol. 18:99-103.

17. Pao, W., et al. 2005. KRAS mutations and primary resistance of lung adenocarcinomas to gefitinib or erlotinib. PLoS. Med. 2:e17.

18. Eberhard, D.A., et al. 2005. Mutations in the epidermal growth factor receptor and in KRAS are predictive and prognostic indicators in patients with non-small-cell lung cancer treated with chemotherapy alone and in combination with erlotinib. J. Clin. Oncol. 23:5900-5909.

19. Arbiser, J.L. 2004. Molecular regulation of angiogenesis and tumorigenesis by signal transduction pathways: evidence of predictable and reproducible 
patterns of synergy in diverse neoplasms. Semin. Cancer Biol. 14:81-91.

20. Cerimele, F., et al. 2005. Reactive oxygen signaling and MAPK activation distinguish Epstein-Barr Virus (EBV)-positive versus EBV-negative Burkitt's lymphoma. Proc. Natl. Acad. Sci. U. S. A. 102:175-179.

21. Govindarajan, B., et al. 2007. Overexpression of Akt converts radial growth melanoma to vertical growth melanoma. J. Clin. Invest. 117:719-729. doi:10.1172/JCI30102.

22. Perry, B.N., et al. 2006. Pharmacologic blockade of angiopoietin-2 Is efficacious against model hemangiomas in mice. J. Invest. Dermatol. 126:2316-2322.

23. Hui, L., Zheng, Y., Yan, Y., Bargonetti, J., and Foster, D.A. 2006. Mutant p53 in MDA-MB-231 breast cancer cells is stabilized by elevated phospholipase $D$ activity and contributes to survival signals generated by phospholipase D. Oncogene. 25:7305-7310.

24. Zheng, Y., et al. 2006. Phospholipase D couples survival and migration signals in stress response of human cancer cells. J. Biol. Chem. 281:15862-15868. 25. Daly, C., et al. 2006. Angiopoietin-2 functions as an autocrine protective factor in stressed endothelial cells. Proc. Natl. Acad. Sci. U. S. A. 103:15491-15496.

26. Hammes, H.P., et al. 2004. Angiopoietin-2 causes pericyte dropout in the normal retina: evidence for involvement in diabetic retinopathy. Diabetes. 53:1104-1110.

27. Tanaka, S., et al. 1999. Biologic significance of angiopoietin-2 expression in human hepatocellular carcinoma. J. Clin. Invest. 103:341-345.

28. Park, J.H., et al. 2007. Serum angiopoietin-2 as a clinical marker for lung cancer. Chest. 132:200-206. 